\title{
Research on English Advertisement Translation based on Relevance Theory
}

\author{
Xiao Li \\ Zhejiang Normal University, Jinhua, Zhejiang, 321004
}

Keywords: Relevance Theory, English, Advertisement Translation

\begin{abstract}
With the progress of society and the development of economy, the increase of international exchange has made the importance of advertising and advertising language translation work further enhanced. In commercial activities, only in line with the audience in the country or region of the language and cultural habits of the slogan translation, in order to accept the majority of advertising audiences accepted. From the perspective of relevance theory, the key to the success of advertising language translation is whether advertising translation can meet the best relevance principle. Based on the basic characteristics of advertising translation, this paper elaborates that the relevance theory can promote the translation of advertisements more directly to the advertising audience and realize the combination of relevance theory and advertisement translation by realizing the best association on the basis of deepening the theory of relevance. - Best relevance. This paper analyzes the influencing factors of advertising language translation from the perspective of relevance theory, and puts forward seven strategies such as literal translation, free translation, transliteration, deletion, imitation, excess and transformation under the guidance of relevance theory. Work to provide more reference and reference.
\end{abstract}

\section{Introduction}

The degree of modernization of social life gradually strengthened, in order to quickly and easily transfer information had to rely on advertising, so people are more and more attention to advertising. The main carrier of advertising is usually language and text, if a commodity advertising as the seller and the buyer's information transmission highway, then the advertising producer is the road builder. How to make advertising for the majority of the audience to accept, how to make advertising more delivery of product information, which is worth thinking about the problem. In the past, most of the relevant research may study the advertising language from the aspects of rhetoric or rhetoric. However, with the deepening of relevant research, the author began to gradually study and study the relevance theory, the author found that the use of relevance theory to advertising Translation analysis is a very meaningful thing. A commodity in the advertising from the initial advertising creative to the production process into reality, and then to the production of the distribution to the circulation of the process, can not do without relevance theory or theoretical point of view, but engaged in the number of research and The results of the formation is not enough, so the author of this writing when the choice of relevance theory through the understanding and application of advertising translation work to conduct a comprehensive, systematic and in-depth analysis to promote the greater development of advertising translation.

\section{Basic Principles of Relevance Theory}

Human beings have a rich and diverse variety of behavior, communication and communication is the information obtained after the handsome selection and optimization made the best decision. When describing a piece of information, then communicate and communicate with a single process. As with the above, the association is the process of processing and combining. If you find the best hypothetical context and proceed with it, you can achieve the best possible communication with the least effort. Good association effect. The contextual effect of the corresponding assumptions on the 
information processing can also be converted, which is the meaning of relevance. The association is an inability to communicate quantification to distinguish the degree of association, and the degree of closeness can only be roughly measured by rough measurement. In other words, there are many categories of assumptions about context, and the selection and selection of these assumptions will give the recipient of the message a different choice and effort. Therefore, when the degree of communication involved in the degree of relevance will be due to the impact of these factors and differences.

How to judge whether the association was carried out? The standard is to achieve the best relevance. In the process of communication and communication, both sides of the communication hope that the information obtained is sufficient and effective, does not contain useless nonsense, that is, the context of the relevant information is simple and effective. The information recipients expect to receive the most useful parts from the various information received and get a reasonable contextual assumption to achieve the best relevance. At the same time, the sender of the information will have the same expectations, otherwise the communication will lose its meaning. Communication is the exchange of information and the realization of the flow of information, the relevant information to optimize and combination, to pseudo-deposit, leaving the most effective information to complete the purpose of communication. Thus Spurs and Wilson define the best association as follows: The stimulus of explicit reasoning is to be sufficiently relevant to the speaker's efforts to process information. And the best association of the information stimulus of the illustrative reasoning can coexist with the communicator's ability and willingness.

At this time, the relationship between the effect of communication and the effort paid is re-placed in front of us, in order to get the best communication effect and pay the least effort, the two sides want to communicate the direction of the other side to make efforts, The party receiving the information will also respond appropriately to the effort of the sender. If the two sides of the information are related, then the communication is simple and convenient. The degree of relevance of information is high, the efforts of both sides will be relatively saved. In order to achieve the most successful communication and communication, we should strive for the best link.

\section{Translation Strategy of Advertising Language from the Perspective of Relevance Theory}

With the increase in the spread of goods between countries and countries, as an important part of the international commodity market communication, people began to pay attention to advertising translation work. Because the enterprise can be based on advertising in the market accounted for decisive factors. In the last chapter we mentioned that a successful ad should make the audience easily get the best contextual effect and then fully understand it. From a cognitive point of view, advertising as a special way of language communication is a communicator or advertiser to use language to promote a product to the reader, to induce or persuade consumers to buy their own products or services. And consumers must use their own inference and cognitive environment to understand the real intention of advertisers. The process described above is consistent with the process of explicit and reasoning about the advertiser's transmission to the consumer's express and expressive intent, and then the consumer draws the conclusion based on the advertiser's explicit reasoning. So the process of advertising translation is also a process of explicit reasoning. Literal translation is a direct and accurate way to convey the original information or feelings of the translation method, this method will keep the source language syntax and rhetoric depends on the language of the text translation norms. In other words, this translation is characterized by its more emphasis on the original text and attempts to achieve the equivalent between the source text and the target text. According to the relevance of the relevant knowledge, literal translation of the way will usually make the initial readers of advertising and advertising target audience to get a consistent cognitive environment. Therefore, if the ad is the most direct way to pass information, then the audience can through the least cognitive efforts to get the core of the commodity information, that is, to get the best contextual effect. In ad translation, literal translation usually applies to the original advertising to convey the information and advertising the real information is consistent and the product does not have any rhetoric or cultural background of the time. 
The original text is consistent, but the situation can change, the translator can play a certain degree of creativity. This translation is relatively more free and flexible, mainly to the target culture and target readers to understand the main. Therefore, despite the reduced loyalty to the original, but the free translation of the target audience easier to understand the ad to achieve the effect of advertising itself. According to the relevance theory, the standard of advertising translation is whether it can achieve its purpose, that is, contextual effect. So a good ad can achieve the desired outcome by looking for the best contextual context of the audience, no matter how failed it is in literal translation. To sum up, the translator can make some adjustments to the free translation to meet the target readers rather than verbatim translation. In this way, the original meaning is that the conversion can be more natural, and thus the translated version is more acceptable. Through the general method as long as the main part of the original or the core part of the appropriate translation, you can let the advertising audience are very easy to find the original and the best link between the translation.

Transliteration is also a translation strategy, it is to replace the original character characteristics, replaced with a new character with relevance. In order to conform to the relevance theory, the transliteration attempts to achieve an optimal replacement for the sound, which can be used primarily to translate the brand name of the advertisement. In the course of translation, the translator needs to make great efforts to infer the original intention of the original or the original meaning of the original brand, and then presented to its target consumers. Therefore, the translator must present the similarity and meaning of the sound through the transliteration, in order to achieve the original and the contextual effect between the translation and deceive the consumer's cognitive environment. Transliteration is usually applied to the language of a country where the language of the language is translated into another country, and it attempts to reacute the voice of the original language. Therefore, the use of transliteration in advertising can retain the original flavor, is an interesting way to attract the interests of consumers. In short, to achieve the best relevance of translation. The translator tries to create a similar sound effect to meet the target audience's communicative intent. As the economy grows and more products are flowing into foreign markets, more and more brand names are translated into English or Chinese transliteration through such strategies.

Imitation is a structural translation method, most of which in the advertising borrow proverbs, proverbs, idioms of the structure, and even the famous advertising language, so that advertising is easier for readers to understand. It is the effective way to advertise it from the theory and practice by imitating some familiar quotations, like poetry, proverbs, saying, imitation, etc. to make up for the original style of loss, structure and image advertising translation. In addition, it can give consumers a strong feeling, so as to promote the sale of advertising products.

Excessive translation, as a translation strategy commonly used in translating text, in other words, appropriate or skilled additions, ie add the need to explain the information for the original text, supplement or comment, thereby enhancing the contextual effect. For example, some information, in the original text is familiar to the source reader, especially the specific information of the culture will be briefly mentioned, however, without being understood by the reader. Therefore, the translator has the responsibility to further expand and explain to the reader, otherwise, the reader will pay too much effort to understand it can not even understand it. According to the theory of relevance translation, the translator should find the context of the original advertisement and the target advertisement so that the target consumer can grasp easily, so the best association of the advertisement intention, the translator must use the translation strategy, Excessive translation of the translation method can help consumers more easily understand the meaning of advertising. Excessive translations can add extra brackets, attributive, synonyms, notes, vocabulary or some other sentences. However, the obvious notes, brackets and vocabulary do not apply to advertising translation, the purpose of advertising is to attract consumers to buy advertising products, which means that consumers do not want to spend too much time to study advertising and read additional annotations. Thus, attributive, synonyms and sentences are mainly used for methods of excess translation. There are two cases of this kind of translation, one that makes some of the key words or 
phrases of deep meaning easier for the target consumer to understand. The second is explained in order to avoid the consumer's understanding of the chaotic specific cultural conditions.

Compared to the original text, you can clearly see the extra words in the source ads did not appear, in addition to "Prairie cigarettes", that is, the translation of the use of excess translation to achieve the intention of the source ads and attract the attention of the target audience. If the source ad is literally translated as "cigarettes made by John Pryor in the UK", the ads for such cigarettes fail because they can not cause the interest and attention of the vast Chinese consumer, nor can they cause them to buy Product desire and can not achieve the purchase behavior. Because the John players are very familiar with the British cigarette manufacturers in the UK, but for Chinese consumers are not familiar with even heard of it. According to the relevance theory, the translator adds some information about the processing of cigarettes, adding tasting and property content in the translation of the text, which will help the Chinese consumer to achieve with the profile to facilitate the best relevance so that the translation produces the same pragmatic effect The

\section{Conclusion}

Social and economic development has been inseparable from the advertising to play the role of information transmission, global integration trend makes advertising inseparable from the translation to achieve cross-regional, cross-language, cross-cultural information exchange function. In the process of advertising translation because of the deposition of years of regional culture and cognitive environment of different, to the translation work has brought difficulty. The relevance theory achieves the best relevance of the context through the explicit reasoning process, which allows the advertising audience to achieve the best correlation through the effort and contextual assumptions in the process of receiving the information, and finally make the translation of the advertising language more Close to the audience where the local cultural characteristics. In this paper, combined with the characteristics of advertising language to prove that the relevance of the theory of advertising on the positive role of translation is effective and obvious. Under the framework of relevance theory, the translation of advertising language can be carried out from the aspects of literal translation, free translation, transliteration, deletion, imitation, excess, transformation and so on, and put forward specific strategies for reference. According to the relevance theory, all of these translation strategies in the advertising application to get enough context effects, in order to achieve the advertising persuasion function. Because of my knowledge and the limitations of the material, this article only on the advertising relevance theory and its translation to explore. Hope it will be advertising from the perspective of relevance theory to study the translation useful, to stimulate the translators to do further research and through efforts to achieve fruitful results.

\section{References}

[1] Li Mingqing. AIDMA law under the ad to observe the aesthetic equivalent [J]. Foreign Language Journal, 2010 (02)

[2] Li Xiaohua, Tang Jun. Context and relevance theory [J]. Journal of Hunan Institute of Humanities and Science, 2008 (04)

[3] Wu Yin. Relevance theory under the cognitive context and advertising language translation [J]. China's high-tech enterprises, 2008 (15)

[4] Chen Shaomin. Relevance theory and translation [J]. Journal of Qiongzhou University. 2007 (06)

[5] Fang Jinxia. Relevance theory of cognitive context [J]. China West Science and Technology, 2006 (25)

[6] Guo Yingzhen. On the relevance of the theory of translation [J]. Journal of Henan Institute of Mechanical and Electrical Engineering, 2005 (06) 